EESTI NSV TEADUSTE AKADEEMIA TOIMETISED, 23. KOIDÉ KEEMIA * GEOLOOGIA. 1974, NR. 3

ИЗВЕСТИЯ АКАДЕМИИ НАУК ЭСТОНСКОИ ССР. ТОМ 23 ХИМИЯ * ГЕОЛОГИЯ. 1974, № 3

УДК $677.14: 543.422: 543.54$

O. KIRRET, E. KULLIK, Lilja LAHE

\title{
OBER DIE IDENTIFIZIERUNG VON BIKOMPONENTFASERN MITTELS DER INFRAROT-SPEKTROSKOPIE UND PYROLYSE-GASCHROMATOGRAPHIE
}

Vor die Analyse der Chemiefaserstoffe werden immer kompliziertere Aufgaben gestellt, insbesondere mit dem Erscheinen neuer modifizierter Fasern und Bikomponentfasern.

Um bessere Verfärbbarkeit, bessere Hygroskopizität und bessere elektrische Eigenschaften der Synthesefasern zu erreichen, werden in die Spinnlösungen (-massen) einige Monomere eingeführt, die verschiedene funktionelle Gruppen haben. In dieser Arbeit werden wir uns mit Untersuchungen einiger Bikomponentfasern und deren Mischungen mittels der Infrarot-Spektroskopie und der Pyrolyse-Gaschromatographie beschäftigen.

Nach Koch [ $\left.{ }^{1}\right]$ : „sind Bikomponentfasern (conjugated fibers) solche Chemiefasern, die aus zwei fest, aber trennbar miteinander chemischen und (oder physikalischen Aufbaues) (z. B. einem Homopolymer und einem modifizierten Copolymer oder zweier artverschiedener Polymeren) schichtig nebeneinander oder umeinander in einer Mischung mit inhomogener Verteilung (Matrix/Fibrillen-System) aufgebaut sind.

Nach ihrem Aufbau lassen sich folgende Typen von Bikomponentfasern unterscheiden:

S/S-Typen - Seite-an-Seite-Typen (Komponenten mit unterschiedlicher Schrumpfneigung nebeneinander eingesponnen) mit „bilatelarer" Struktur wie bei Schafwolle (side-by-side)

C/C-Typen - konzentrisch oder exzentrisch aufgebaute Hülle/KernTypen (Komponenten umeinander eingesponnen) (centric covercore)

M/F-Typen - Matrix/Fibrillen-Typen (auch „Bikonstuentenfasern” genannt) (matrix-fibril)".

Wie aus dem Aufbau der Bikomponentiasern (man spricht bereits von Komponentfasern) zu ersehen, werden die Erkennungs-, Trennungs- und Bestimmungsmethoden immer komplizierter.

Der Untersuchung wurden folgende Chemiefasern und deren Mischungen unterzogen:
1. Nitron
2. Orlon 21
3. Orlon 23
- Polyacrylfaser
- Polyacrylnitril-Bikomponentfaser Typ S/S
3. Orlon 23
- Polyacrylnitril-Bikomponentfaser Typ S/S $\mathrm{PAC} /$ modif. PAC

4. Orlon $21 /$ Nitron Mischung 1:1 

5. Vinylon
6. Thermovyl
7. Cordela
8. Thermovyl/Vinylon Mischung 1:1

- Polyvinylalkoholfaser

- Polyvinylchloridfaser
- Polyvinylchlorid/Polyvinylalkohol-Bikompo- nentfaser Typ S/S

Die IR-Spektren wurden dabei mit UR-10 aufgenommen (Abb. 1). Es wurde dabei $\mathrm{KBr}$-Tabletten Methodik benutzt.

Tabelle 1

Elementaranalyse der untersuchten Chemiefasern (\%)

\begin{tabular}{l|c|c|c}
\hline & C & H & N \\
\hline Nitron & 67.23 & 5.64 & 24.22 \\
Orlon 21 & 65.88 & 5.70 & 24.76 \\
Orlon 23 & 66.10 & 5.57 & 24.51 \\
Thermovyl & 38.39 & 4.80 & - \\
Vinylon & 56.18 & 8.91 & - \\
Cordela & 49.66 & 7.36 & -
\end{tabular}

Zur Elementaranalyse wurde ein Analysator Modell 185, der F-a Hewlett-Packard benutzt.

Analyseneinwaage: $0,7 \mathrm{mg}$.

Das in den IR-Spektren von Orlon Fasern (Tabelle 2) vorhandene Absorptionsmaximum von $1667 \mathrm{~cm}^{-1}$ kann von einem disubstituierten Amidzusatz sein, zum Beispiel Vinylpyrrolidon [ $\left.{ }^{2}\right]$.

Tabelle 2

Charakteristische Absorptionsbänder der IR-Spektren der Polyacrylnitrilfaserstoffe (in $\mathrm{cm}^{-1}$ )

\begin{tabular}{|c|c|c|c|c|}
\hline Nitron & $\begin{array}{l}\text { Orlon } 21 \\
\text { PAC }\end{array}$ & $\begin{array}{l}\text { Orlon 21/ } \\
\text { Nitron }\end{array}$ & $\begin{array}{c}\text { Orlon } 23 \\
\text { PAC } / \text { mod. PAC }\end{array}$ & Zuordnung \\
\hline & & & 772 & $v(\mathrm{C}-\mathrm{CN}) ; \mathrm{t}\left(\mathrm{CH}_{2}\right)$ \\
\hline & $\begin{array}{l}1009 \\
1007\end{array}$ & $\begin{array}{l}1005 \\
1035\end{array}$ & & \\
\hline 1070 & & 1065 & 1069 & $\begin{array}{c}v_{\mathrm{s}}(\mathrm{C}-\mathrm{C}) ; v\left(\mathrm{CH}_{2}\right) \\
\delta(\mathrm{C}-\mathrm{C}-\mathrm{CN})\end{array}$ \\
\hline 1170 & 1107 & & 1174 & \\
\hline & $1194-1244$ & $1165-1240$ & $1200-1260$ & $\begin{array}{l}v(\mathrm{C}-\mathrm{C}) ; \mathrm{w}(\mathrm{CH}) ; v(\mathrm{C}-\mathrm{O}) ; \\
\text { w }\left(\mathrm{CH}_{2}\right)\end{array}$ \\
\hline $\begin{array}{l}1360 \\
1365 \\
1455\end{array}$ & $\begin{array}{l}1369 \\
1460\end{array}$ & \multirow{4}{*}{$\begin{array}{l}1360 \\
1455 \\
1665 \\
1730 \\
2237 \\
2860 \\
2915 \text { sh } \\
2930\end{array}$} & \multirow{4}{*}{$\begin{array}{c}1460 \\
1675 \\
1727 \\
2235 \\
2850-2885 \\
2920 \\
2930 \\
3460\end{array}$} & $v_{\mathrm{a}}(\mathrm{C}-\mathrm{C}) ; \delta(\mathrm{CH}) ; \mathrm{w}\left(\mathrm{CH}_{2}\right)$ \\
\hline 1730 & 1670 & & & \\
\hline $\begin{array}{l}2240 \\
2860\end{array}$ & $\begin{array}{l}2235 \\
2872\end{array}$ & & & $\begin{array}{l}v(\mathrm{CN}) \\
v_{\mathrm{s}}\left(\mathrm{CH}_{2}\right)\end{array}$ \\
\hline 2930 & 2932 & & & $\begin{array}{l}v(\mathrm{CH}) \\
2 \times v(\mathrm{C}=\mathrm{O})\end{array}$ \\
\hline
\end{tabular}

Ein Absorptionsmaximum von $1730 \mathrm{~cm}^{-1}$ läßt ein esterartiges Comonomer erwarten. Bei Nitronfasern könnte man mit Methylacrylat- oder 

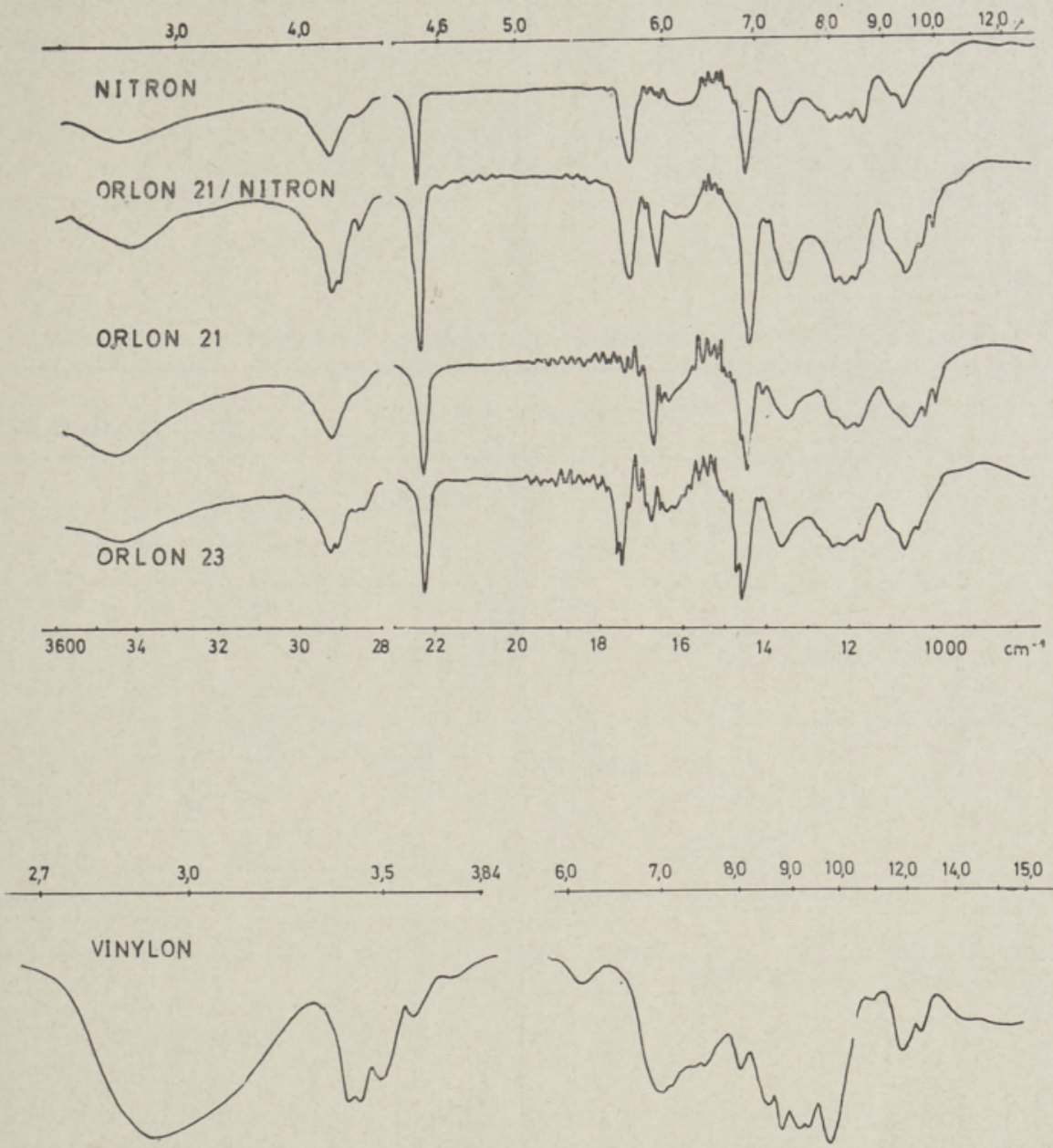

\section{THERMOVYL}

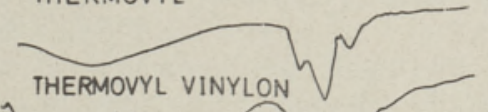

$1: 1$

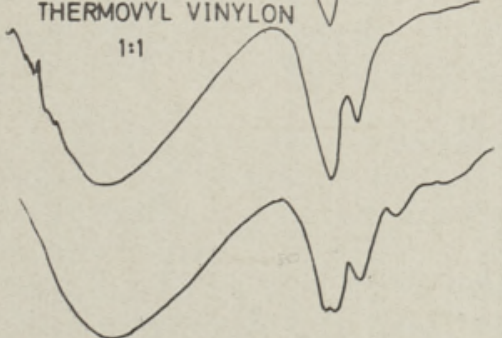

CORDELA
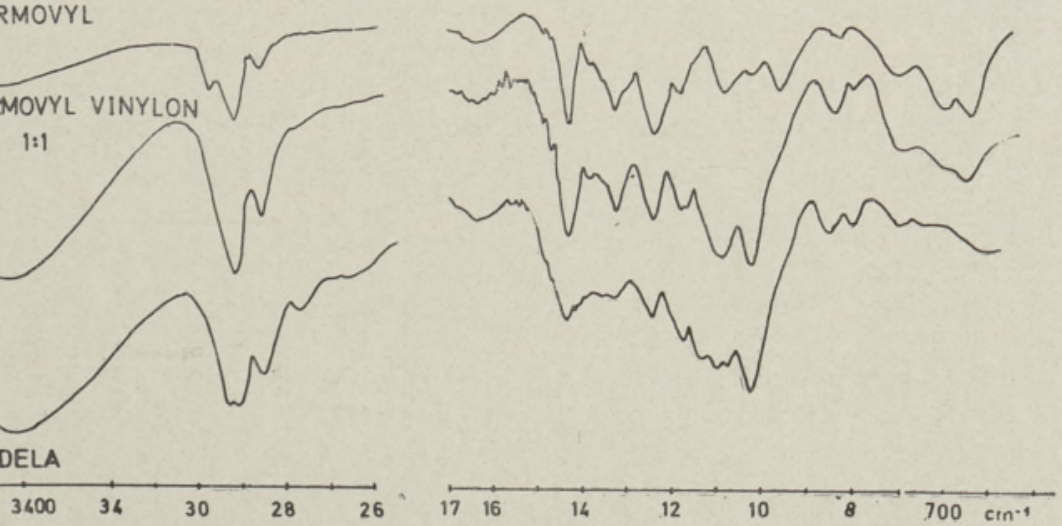

Abb. 1. Infrarot-Spektren von Polyacrylnitril-, Bikomponent-, Polyvinylchlorid-, Polyvinylalkoholfasern und deren Mischungen. 


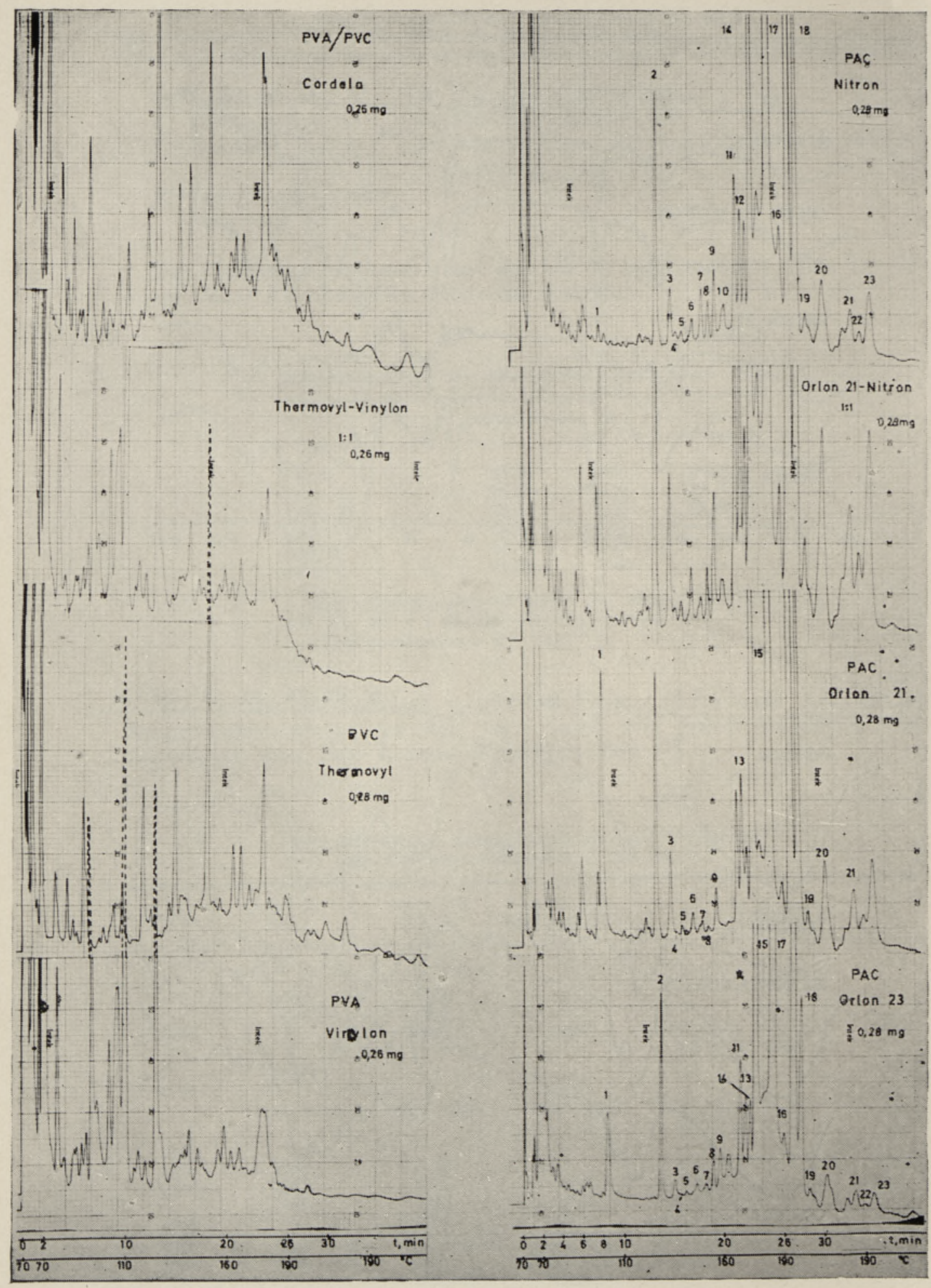

Abb. 2. Chromatogramme von Bikomponent-, Polyacrylnitril-, Polyvinylchlorid-, Polyvinylalkoholfasern und deren Mischungen. 
Vinylacetatzusätzen rechnen; da aber in IR-Spektren das für die Azetate charakteristische Absorptionsband von $1235 \mathrm{~cm}^{-1}$ fehlt, haben wir es wahrscheinlich mit einem Methylacrylat-Zusatz zu tun.

Das IR-Spektrum von Vinylon als Polyvinylalkohol enthält die den sekundären Alkoholen entsprechende Valenzschwingung von $1075 \mathrm{~cm}^{-1}$ und das den Polyassoziaten entsprechende breite flache Maximum im Bereich von 3340 bis zu $3480 \mathrm{~cm}^{-1}$ (Tabelle 3 ). Das für die Polyvinyl-

Tabelle 3

Charakteristische Absorptionsbänder der IR-Spektren der Chemiefaserstoffe

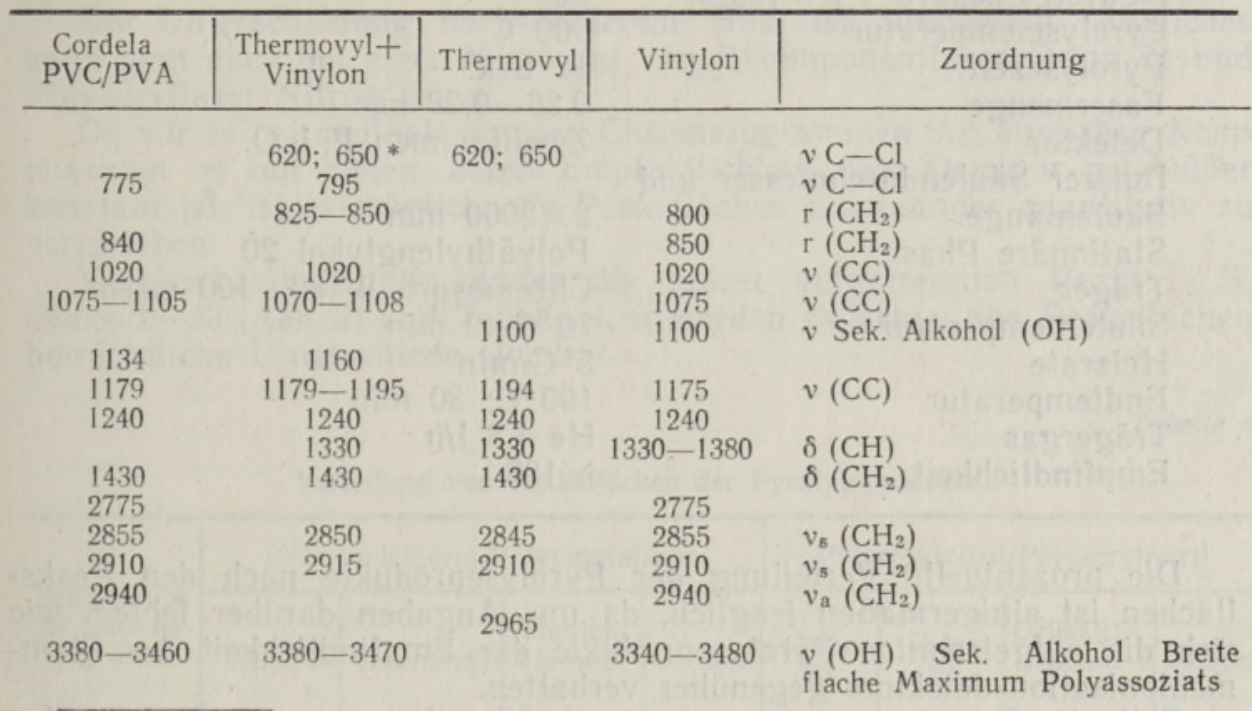

* Die Schwingungen der $\mathrm{C}-\mathrm{Cl}$ Bindungen sind sehr schwach.

alkohole charakteristische Bereich ist auch der Bikomponentfaser Cordela eigen. Den Polyvinylchloriden charakteristische $\mathrm{C}-\mathrm{Cl}$ u. a. Schwingungen kommen bei den IR-Spektren von Thermovyl und ThermovylVinylon Fasermischungen in Erscheinung.

Beim Vergleichen der Angaben der Elementaranalyse und der Infrarotspektren einerseits und der Pyrolyse-Chromatogramme anderseits ist ersichtlich, da $B$ die Chromatogramme mehr Information enthalten (Abb. 2).

Gleichzeitig kann man bemerken, daB die Infrarotspektren und die mikroanalytischen Angaben leichter reproduzierbar sind als die pyrolyse-gaschromatographischen Daten, die von verschiedenen Bedingungen abhängig sind.

Bei der Pyrolyse von verschiedenen modifizierten Faserstoffen derselben Klassen entstehen in den Pyrolyseprodukten qualitative und quantitative Veränderungen. Im allgemeinen könnte man die Bikomponentfaser als Fasermischung oder eine Mischung von verschiedenen Faserstoffmodifikationen betrachten.

Vom Standpunkt der qualitativen Analyse lassen sich diejenigen Bikomponentfasern leichter unterscheiden, die ihrem chemischen Aufbau nach merklich unterschiedlich sind. Hier kann man nach den unterschiedlichen Retentionszeiten der Peaks verhältnismäßig leicht die Pyrolyseprodukte erkennen oder zuordnen. 
Ist die Bikomponentfaser aus Polymeren hergestellt, die im chemischen Aufbau nur wenig voneinander unterschiedlich sind, so ist die qualitative Analyse ziemlich schwierig.

Für die Pyrolyse-Chromatographie wurde ein Perkin Elmer Gaschromatograph Mod. 900 mit einem Integrator 3370 A (F-a Hewlett Packard) benutzt.

\section{Arbeitsbedingungen des Gaschromatographen:}

Perkin-Elmer

Hewlett-Packard Pyrolysator

Pyrolysetemperatur

Pyrolysezeit

Fasermenge

Detektor

Innerer Säulendurchmesser und

Säulenlänge

Stationäre Phase

Träger

Säulentemperatur

Heizrate

Endtemperatur

Trägergas

Empfindlichkeit
900

90

$900^{\circ} \mathrm{C}$

12 Sek.

$0,26-0,28 \mathrm{mg}$

Zweiflammen F. I. O.

$2 \times 2000 \mathrm{~mm}$

Polyäthylenglykol 20

Chromosorb W 80-100 mesh

$70^{\circ} \mathrm{C} ; 2 \mathrm{~min}$

$5^{\circ} \mathrm{C} / \mathrm{min}$

$190^{\circ} \mathrm{C} ; 30 \mathrm{~min}$

He $1.81 / \mathrm{h}$

$\times 128$

Die prozentuelle Verteilung der Pyrolyseprodukte nach den Peaksflächen ist einigermaßen fraglich, da uns Angaben darüber fehlen, wie sich die aufgetrennten Pyrolyseprodukte der Empfindlichkeit des Flammenionisationsdetektors gegenüber verhalten.

Falls die Chromatogramme nur für die Identifizierung von Faserstoffen benutzt werden, braucht man nicht immer jeden Peak zu identifizieren.

Ein Oberblick über die Verteilung der Pyrolyseprodukte nach den Peaksflächen ist aus Tabelle 4 ersichtlich.

Den Flächen nach gehören die Peaks meistens in die Gruppe der Größenordnung von $1-0,01 \%$.

Peaks, die eine Fläche von mehr als $10 \%$ der Gesamtfläche haben, gibt es zwei bis drei. Eine Identifizierung mit Hilfe solcher breitgrenziger Verteilung ist unmöglich. Nimmt man zur Analyse größere Probemengen, so verschlechtert sich die Trennung der einzelnen Peaks, wodurch die Identifizierung noch komplizierter wird. Bei der Vergrößerung der Empfindlichkeit des Gaschromatographen kommen wiederum verschiedene Störungen vor, welche die genaue Bestimmung der Peaksflächen unmöglich machen.

Wie aus den Chromatogrammen der Pyrolyseprodukte ersichtlich ist (Abb. 1), kann man sehr einfach solche Bikomponentfasern identifizieren, deren chemische Aufbausubstanzen verschieden sind.

Das Chromatogramm widerspiegelt die spezifischen Pyrolyseprodukte, die bei der thermischen Zersetzung zweikomponentiger Polymeren (PVC und PVA 1:1) entstanden sind.

Untersucht man näher Chromatogramme, die bei der Pyrolyse von Chemiefasermischungen oder Bikomponentfasern entstanden sind, so werden folgende Anderungen sichtbar:

1) Unterschiede in den Pyrolyseprodukten von einer Chemiefaser oder Bikomponentfaser sind durch das Chromatogramm feststellbar. 
2) In den Chromatogrammen der Pyrolyseprodukte von Fasermischungen kommen nicht den Ausgangspolymeren eigene Komponenten in Erscheinung.

3) In den Pyrolyseprodukten von Chemiefasermischungen oder Bikomponentfasern sind neue individuelle Verbindungen entstanden.

Das Vorhandensein solcher Peaks erleichtert und erweitert den Bestimmungsumfang der Polymere.

Alle diese Fälle kommen in den betrachteten Systemen mehr oder weniger in Erscheinung.

In den Chromatogrammen Orlon 21 und 23 sind den Polyacrylfasern entsprechende Peaks zu sehen.

Die Unterscheidung nach äußerem Bild ist unmöglich. Ahnliches kann man auch bei einer Mischung von Bikomponentfaser Orlon 21 und Polyacrylfaser Nitron sagen.

$\mathrm{Da}$ wir es bei den betrachteten Chromatogrammen mit ähnlichen Komponenten zu tun haben, deren Empfindlichkeit dem Detektor gegenüber konstant ist, ist es möglich, die Peaksflächen miteinander quantitativ zu vergleichen.

Vergleicht man miteinander die besser aufgetrennten Peaks, z. B. Peaks 1-23 (Abb. 2) und Tabelle 4, so werden zwischen den Peaksflächen beträchtliche Unterschiede sichtbar.

Tabelle 4

Verteilung von Peaksflächen der Pyrolyseprodukte

\begin{tabular}{|c|c|c|c|c|c|c|c|c|}
\hline \multirow[b]{2}{*}{$\begin{array}{c}\text { Peaksfläche } \\
\text { auf Chroma- } \\
\text { togramm }\end{array}$} & \multicolumn{4}{|c|}{ Polyvinylchlorid/Polyvinylalkohol } & \multicolumn{4}{|c|}{ Polyacrylnitril/Polyacrylnitril } \\
\hline & Vinylon & Thermovyl & $\begin{array}{l}\text { Vinylon/ } \\
\text { Thermo- } \\
\text { vyl 1:1 }\end{array}$ & Cordela & $\begin{array}{c}\text { Orlon } \\
23\end{array}$ & $\begin{array}{c}\text { Orlon } \\
21\end{array}$ & $\begin{array}{c}\text { Orlon } 21 / \\
\text { Nitron } \\
1: 1\end{array}$ & Nitron \\
\hline
\end{tabular}

\begin{tabular}{crrrrrrrr}
$<10$ & 3 & 2 & 4 & 2 & 3 & 2 & 3 & 3 \\
$10-1$ & 9 & 14 & 9 & 15 & 5 & 5 & 7 & 9 \\
$1-0,1$ & 16 & 15 & 15 & 20 & 11 & 11 & 18 & 20 \\
$0,1-0,01$ & 8 & 6 & 11 & 12 & 17 & 18 & 17 & 14 \\
$>0,01$ & 2 & 5 & 2 & 1 & 7 & 7 & 4 & 6 \\
\hline Peakszahl & 38 & 42 & 41 & 50 & 43 & 43 & 49 & 52
\end{tabular}

Größere Unterschiede lassen sich bei den für die Polyacrylnitrilfasern charakteristischen Peaks 14-18 feststellen, wodurch es möglich wird, auf Grund bestimmter Verhältnisse zwischen den Peaks über die Zugehörigkeit der Fasern zu gewissen Faserstoffklassen zu urteilen.

Bei der Analyse von Polyacrylnitril-Bikomponentfasern muß in jedem Einzelfall mit den unterschiedlichen Peaksflächen gerechnet werden.

\section{I T ER A T UR}

1. Koch P.-A., Textil-Industrie, 72, 253(1970)

2. Hummel D., Scholl Fr., Atlas der Kunststoffanalyse, Carl Hanser Verlag, München Verlag Chemie GmbH, Weinheim/Bergstraße, I, Teil 1, 188 (1969).

Institut für Chemie

der Akademie der Wissenschaften der Estnischen SSR
Eingegangen am 22. März 1974 
O. KIRRET, E. KULLIK, Lilja LAHE

\section{BIKOMPONENTKIUDUDE IDENTIFITSEERIMISEST INFRAPUNASE SPEKTROSKOOPIA JA PUROLUUSI-GAASIKROMATOGRAAFIA ABIL}

Tehti kindlaks, et keemiliste kiudude resp. bikomponentkiudude infrapunastes spektrites esinevate karakteersete absorptsiooniribade körval esineb ka absorptsiooniribasid, mis iseloomustavad ühe või teise bikomponentkiu formeerimisel kasutatud lisandeid. Bikomponentkiudude ja etalooniks võetud keemiliste kiudude pürolüüsil saadud kromatogrammide võrdlemine vōimaldab määrata bikomponentkiudude kuuluvuse teatud keemiliste kiudude klassi.

\section{О. КИРРЕТ, Э. КЮЛЛИК, Лилья ЛАХЕ}

\section{ОБ ИДЕНТИФИКАЦИИ БИКОМПОНЕНТНЫХ ВОЛОКОН МЕТОДАМИ ИНФРАКРАСНОИ СПЕКТРОСКОПИИ И ПИРОЛИЗНОИ ГАЗОВОИ ХРОМАТОГРАФИИ}

Исследованы возможности применения методов инфракрасной спектроскопии и пиролизной газовой хроматографин для идентификации бикомпонентных волокон.

Показано, что в ИК-спектрах наряду с характерными абсорбционными полосами имеются полосы, характеризующие те добавки, которые были использованы при образовании бикомпонентных волокон. При сравнении хроматограмм, полученных при пиролизе эталонных химических и бикампонентных волокон, можно определить класс, куда принадлежат последние. 